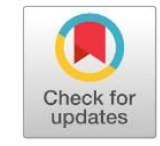

Received 01 ${ }^{\text {th }}$ October 2019 Accepted $24^{\mathrm{d}}$ February 2019 www.ijpefs.com

\title{
Systematic Review on the Associations between Objectively Measured Breaks in Sitting Time and Cardiovascular Health in Youth
}

\author{
Sousa-Sá E. a,e,*, McNeill, J. a, Pereira, JR a,c , Zhang, Z a , Okely, AD.a,e, Santos, R.a,b,d \\ a Early Start, Faculty of Social Sciences; University of Wollongong, Wollongong, NSW \\ 2522, Australia. \\ b Research Centre in Physical Activity, Health and Leisure, University of Porto, Portugal. \\ c Research Unit for Sport and Physical Activity, University of Coimbra, Coimbra, Portugal. \\ d Universidade Lusófona de Humanidades e Tecnologia, Lisboa, Portugal. \\ e Illawarra Health and Medical Research Institute, Wollongong, NSW, Australia. \\ *Corresponding Author Email: emdsr885@uowmail.edu.au
}

\begin{abstract}
The aim of this systematic review was to summarize the literature on the associations between breaks in sitting time and cardiovascular health, in children and adolescents. The search was conducted using five databases (MEDLINE, SCOPUS, WEB OF SCIENCE, PSYCINFO and CINAHL) through to 01 October 2019. Due to heterogeneity of the data, meta-analyses were not possible. We screened 2577 studies, and 15 studies were included, representing 9116 participants, from six different countries. Five observational studies and four experimental studies showed associations between breaks in sitting time and cardiovascular health, i.e. an increased number of breaks in sitting time was negatively associated with a cardiovascular health outcome. No associations between number of breaks in sitting time and cardiovascular health outcomes were found in the six remaining studies. Studies examining associations between breaks in sitting time and cardiovascular health in children have shown some favorable associations. More epidemiological evidence is required, to inform lifestyle interventions and public health policies, which could translate into long-term implications on population health.
\end{abstract}

Key Words: Cardiovascular risk factors, Sedentary time, Youth; Body composition, Cardiometabolic health, Pediatric

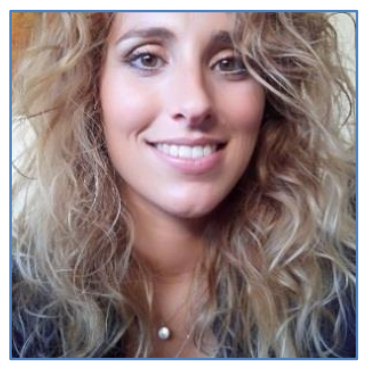

Dr. Eduarda Sousa-Sá

Diseases, Sedentary Behavior, Sitting Time, Physical completed her PhD in Physical Activity, Executive functions, Sleep and Movement Activity and Public Health, at Combination. Currently she is project manager of the the Early Start - Faculty of Portuguese 24h Movement Guidelines project.

Social Sciences, University of

Wollongong, Australia in May 2016-2019 funded by an International Postgraduate Tuition Award from the University of Wollongong and a Discovery Early Career Research Award from the Australian Research Council from her supervisor. Dr Sousa-Sá was awarded in July 2019 with a postdoctoral fellowship at the University of Minho - Portugal, funded by the Calouste Gulbenkian Foundation. Her research interests include Public Health, Cardiovascular

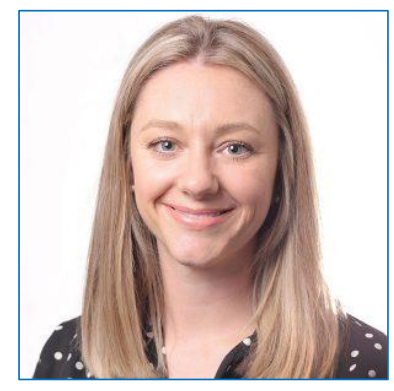

Dr. Jade McNeill completed her PhD in area of physical activity and screen time in early childhood, at the Early Start - Faculty of Social Sciences, University of Wollongong, Australia in 2015-2019, and was funded by an International Postgraduate Tuition Award from the University of Wollongong and a Discovery Early 


\section{Sousa-Sá E et al.,/2020}

Career Research Award from the Australian Research Council from her supervisor. Dr McNeill is currently at UNSW working in translational health research within early childhood. Her research interests are physical activity, screen time, sleep, cognitive and psychosocial development and translational research and movement behaviors.

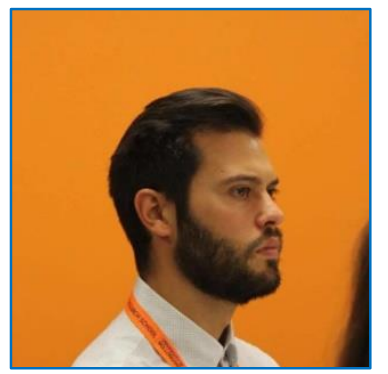

João Pereira is currently finalizing his $\mathrm{PhD}$ in the area of sedentary behavior in young children, at the Early Start - Faculty of Social Sciences, University of Wollongong, Australia funded by an International

Postgraduate Tuition Award from the University of Wollongong. His research interest is in movement behaviors, particularly sedentary behavior.

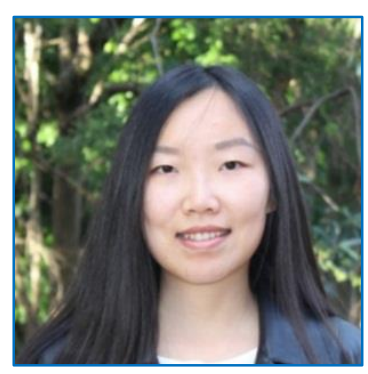

Dr. Zhiguang Zhang completed her PhD in area of childcare environment, 24hour movement behavior and adiposity in young children at the Early Start - Faculty of Social Sciences, University of Wollongong, Australia in December, 2019 with a scholarship from the China Scholarship Council and an International Postgraduate Tuition Award from the University of Wollongong. Dr Zhang was recently awarded with a postdoctoral fellowship at the University of Alberta. Her research interests are environment, movement behavior and health in children.

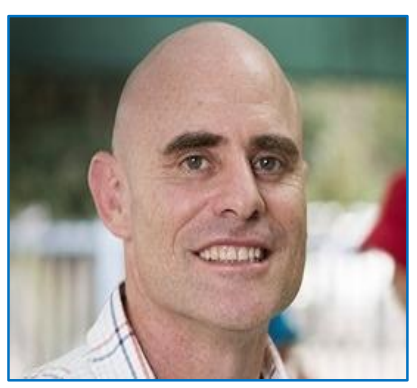

Dr. Anthony Okely is a Full Professor in the Faculty of Social Sciences and Director of Research at Early Start at the University of Wollongong, Australia. He has been awarded over \$14 Million in competitive funding and published around 250 peer-reviewed journal articles that have been cited more than 15,000 times. Dr Okely's research focuses on movement behaviors (physical activity, sedentary behavior, and sleep) in children. He is the 7th most published researcher in the world on physical activity and children. He led the research team that developed and recently updated the Australian 24-hr Movement Guidelines for Children 0-5 years, and the Australian 24-hr Movement Guidelines for Children and Young People. He was also part of the Guideline Development Group for the WHO guidelines on physical activity, sedentary and sleep behaviors in children under 5 years of age, South African 24-hr Movement Guidelines for Birth to 5 Years, and UK Physical Activity Guidelines for children under 5. He is current leading an international surveillance study of 24-hour movement behaviors in the early years (called SUNRISE) which involves around 30 countries, two-thirds of which are either low- or middle-income according to the UN Human Development Index.

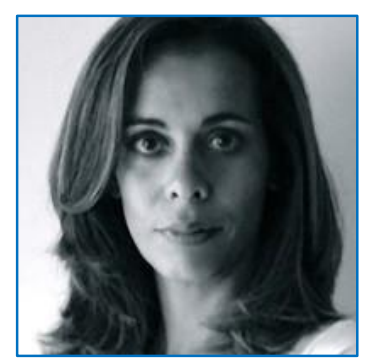

Dr, Rute Santos completed her PhD in Physical Activity and Health in 2009, at the Research Centre in Physical Activity, Health and Leisure at the University of Porto, Portugal with a grant from the Portuguese Foundation for Science and Technology. In 2011 she concluded a postdoctoral fellowship in this same research center. In 2015 she obtained the title of "Agregada"/Tenure in Physical Activity and Health by the University of Porto. From 2015 to 2018 she was granted a Discovery Early Career Research Award from the Australian Research Council, at the University of Wollongong, Australia. Currently, Dr Santos is a Professorial Researcher at University of Porto and Deputy-director of the National Physical Activity Promotion Program of the Portuguese Directorate-general of Health. She is a Movement Behavior Epidemiologist, has expertise on health-related associations of sleep, sedentary behavior and physical activity. Relevant projects as principal investigator include the Portuguese $24 \mathrm{~h}$ Movement Guidelines; the Australian 24h Movement Guidelines; the Get UP! Study and the LabMed Study; these were ground-breaking projects that greatly advance Movement Behavior knowledge. 


\section{Sousa-Sá E et al.,/2020}

\section{Introduction}

Sedentary time is defined as the time spent for any duration (e.g., minutes per day) or in any context (e.g., at school or work) in sedentary behaviors [1]. Within sedentary time, sitting is defined as a type of sedentary behavior, characterized by a position in which one's weight is supported by one's buttocks rather than one's feet, and in which one's back is upright. A sedentary break is defined as a non-sedentary bout in between two sedentary bouts, being that a sedentary bout is a period of uninterrupted sedentary time [1].

Cardiovascular disease is the largest cause of death among adults worldwide [2] and there is emerging evidence showing that cardiovascular risk factors and lifestyle behaviors tend to track from childhood into adulthood, contributing to the risk of diseases decades later[3, 4]. Indeed, as sedentary time seems to be a prevalent behavior that may contribute to cardiovascular risk in young children $[5,6]$, public health recommendations encourage young children and youth to limit their sedentary time. Canadian and Australian 24-Hour Movement Guidelines for the Early Years recommend that children younger than 1 year old should not be restrained for more than 1 hour at a time and screen time is not recommended; for toddlers, the recommendation is similar with the remark that for those younger than 2 years old, sedentary screen time is not recommended and, for those aged 2 to 4 years old, sedentary screen time should be no more than 1 hour [7, 8]. The Canadian 24-Hour Movement Guidelines for Children and Youth recommend that children between 5 and 17 years old should have no more than 2 hours per day of recreational screen time and should limit sitting for extended periods [8]. In this line of thought, it seems important to understand what the literature says about the relationship between breaking up sedentary time/sitting time and cardiovascular health, in children, to prevent having an adult population with low health levels.

Epidemiological studies have described negative associations between total sedentary time and cardiovascular risk factors, in children and adolescents $[6,5]$, suggesting that prolonged sitting may have a quantifiable health effect in this population. On the other hand, evidence also shows that breaking-up prolonged sitting time with lightintensity physical activity(LPA) can be beneficial for adults' cardio-metabolic health [9-12], and is associated with decreased all-cause mortality risk [13]. However, it is yet unclear which features and patterns of sedentary time might be most strongly associated with increased health risk, in children and adolescents $[6,5]$. The two most recent reviews on the sedentary behaviors and health indicators were unable to draw conclusions on the associations between breaks in sitting time and health outcomes. Poitras and colleagues' review [5] in young children (0-4 years) did not assess the effects of breaks in sitting time and health outcomes, and Carson and colleagues' review [6] showed that in youth (5-17 years) breaking up sitting time was not consistently associated with any health outcomes.

In this context, the aim of this systematic review was to summarize the literature on the associations between breaks in sitting time and cardiovascular health, in children and adolescents aged 0 to 18 years. Such review is warranted, given the increased research interest on this topic, in the last years. The evidence herein provided has the potential to inform future lifestyle interventions and public health policies and guidelines.

\section{Method}

\subsection{Protocol and Registration}

This review was registered with the international prospective register of systematic reviews PROSPERO network, registration no. CRD42018083790. We followed the PRISMA statement for reporting systematic reviews and meta-analyses [14].

\subsection{Search strategy and data sources}

A structured computer-based search on the electronic databases MEDLINE, SCOPUS, WEB OF SCIENCE, PSYCINFO and CINAHL, on peer-reviewed articles was performed by one author (ES), through to 01 October, 2019 (including all available years because of the recent interest to investigate breaks 


\section{Sousa-Sá E et al.,/2020}

in sitting time). The search strings used can be seen in supplementary table 1.

Studies were imported into Reference Manager Software (EndNote X8) where duplicate records were removed. Titles and abstracts were then reviewed and checked for relevance, and pertinent full text articles were obtained and assessed for inclusion by four authors (ES, JM, JP and ZZ). Reference lists of included articles and relevant reviews were also checked for additional relevant studies. Disagreements $(n=2)$ were resolved by consensus with two other authors (RS and ADO).

\subsection{Study selection criteria}

To be included, studies had to be published in English, Portuguese, French, Spanish or Chinese. The study participants were apparently healthy but included overweight and/or obese children and youth (from birth to 18 years old).

There were no limitations on study designs. However, methods papers, conference abstracts or proceedings, editorials, commentaries and reviews were not considered. For longitudinal studies, the age criteria applied to, at least, one exposure measurement in time. Articles had to meet the $a$ priori determined population, intervention, comparator, and outcome (PICO) study criteria [15]. For intervention studies, the considered exposure was measures of breaks in sitting time. A comparator or control group was not required.

Breaks in sitting time had to be measured objectively (e.g., pedometer, accelerometer, inclinometer, direct observation). Therefore, studies were required to have a measure of breaks in sitting time per se, rather than just a measure of the number of bouts of sitting time. For the outcomes, changes or measures in children's cardiovascular health outcomes had to be reported. The studies needed to contain a measure of cardiovascular health as a dependent variable and had to assess the associations between breaks in sitting time and cardiovascular health outcomes.

\subsection{Data extraction and synthesis}

Descriptive study characteristics, exposure, outcome, validity/feasibility of tools used for assessments, and results information were extracted for each study, through a coding framework to record information on a variety of details on two tables. Table 1 shows the characteristics of the studies included, for both observational and experimental designs, reporting also on assessment of the outcome, type of statistical analysis with confounder, intervention details and main results.

\subsection{Quality assessment}

Four authors (ES, JM, JP and ZZ) independently assessed the risk of bias (ROB) using GRADE framework (Grading of Recommendations Assessment, Development, and Evaluation)[16]. GRADE does not have an official tool to assess ROB in observational/experimental studies but recommends the types of features to examine [17]. General quality of evidence was assessed for each included study design based on five characteristics: (i) selection bias, (ii) attrition bias, (iii) detection bias, (iv) performance bias and (v) selective reporting bias. To be in line with the GRADE framework, quality of evidence scores start at "high" for randomized controlled trials and "low" for all other experimental and observational studies. Additional information on GRADE methodology can be found elsewhere [16] and details for each criteria used to assess ROB can be seen in table 2 .

\subsection{Data analysis}

Meta-analyses were not possible due to insufficient homogeneity of statistical, clinical and methodological characteristics of the data. Therefore, we conducted a qualitative synthesis, with all studies weighted equally and the results presented narratively. Statistical significant of included studies was deemed based on what each study reported to be significant. 
Sousa-Sá E et al.,/2020

Table 1. Characteristics of the included studies, divided by observational and experimental design (in chronological order)

\begin{tabular}{|c|c|c|c|c|c|c|c|}
\hline $\begin{array}{l}\text { Author } \\
\text { (Study) }\end{array}$ & Design & Study sample & $\begin{array}{l}\text { Measurement of } \\
\text { breaks in sitting } \\
\text { time (exposure) }\end{array}$ & Breaks unit & $\begin{array}{c}\text { Assessment of } \\
\text { cardiovascular } \\
\text { outcomes }\end{array}$ & $\begin{array}{l}\text { Statistical analysis \& } \\
\text { Confounders }\end{array}$ & Main results \\
\hline \multicolumn{8}{|c|}{ Observational studies } \\
\hline $\begin{array}{l}\text { Carson \& } \\
\text { Janssen, 2011 } \\
\text { (Nutrition } \\
\text { Health and } \\
\text { Nutrition } \\
\text { Examination } \\
\text { Survey - } \\
\text { NHANES) [27] }\end{array}$ & $\begin{array}{c}\text { Cross- } \\
\text { sectional }\end{array}$ & $\begin{array}{c}\text { USA: } 2527 ; \\
\text { mean age }=13 \\
(10-16) \text { years } \\
(49.2 \% \text { girls })\end{array}$ & $\begin{array}{l}\text { Actigraph AM- } \\
7124 \\
\text { accelerometer } \\
\quad \text { (uniaxial) }\end{array}$ & $\begin{array}{l}\text { Breaks in sedentary time } \\
\text { were defined within each } \\
\text { bout of SB, as the } \\
\text { minutes equal to low } \\
\text { intensity activity or } \\
\text { MVPA. }\end{array}$ & $\begin{array}{l}\text { Age and sex-adjusted } \\
\text { cardiometabolic risk } \\
\text { score (WC, SBP, non- } \\
\text { HDL cholesterol, and } \\
\text { C-reactive protein). }\end{array}$ & $\begin{array}{l}\text { Logistic regression } \\
\text { analyses } \\
\text { Confounders: Age, gender, } \\
\text { ethnicity, SES, smoking, } \\
\text { total fat, saturated fat, } \\
\text { dietary cholesterol, } \\
\text { sodium, volume of SB and } \\
\text { MVPA. }\end{array}$ & $\begin{array}{l}\text { The prevalence of high cardio- } \\
\text { metabolic risk score did not vary } \\
\text { across quartiles of the breaks in } \\
\text { bouts of SB measures ( } p_{\text {trend }}=0.78 \text { ). } \\
\text { Breaks in bouts of SB measures did } \\
\text { not predict ( } p_{\text {trend }}=0.67 \text { ) high } \\
\text { cardio-metabolic risk score after } \\
\text { adjusting for various confounders, } \\
\text { the volume of SB and MVPA. }\end{array}$ \\
\hline $\begin{array}{l}\text { Colley et al., } \\
2013 \\
\text { The Canadian } \\
\text { Health } \\
\text { Measures } \\
\text { Survey (CHMS) } \\
{[20]}\end{array}$ & $\begin{array}{c}\text { Cross- } \\
\text { sectional }\end{array}$ & $\begin{array}{l}\text { Canada: } 1608 ; \\
\text { mean } \\
\text { age }=11.6 \pm 1.1 \\
\text { years } \\
\text { (49.7\% girls) }\end{array}$ & $\begin{array}{c}\text { Actical } \\
\text { accelerometer }\end{array}$ & $\begin{array}{l}\text { Breaks in sedentary time } \\
\text { were defined as an } \\
\text { interruption in } \\
\text { sedentary time (lasting a } \\
\text { minimum of one minute) } \\
\text { in which there was a } \\
\text { transition in } \\
\text { accelerometer count } \\
\text { from }<100 \mathrm{cpm} \text { to } \geq 100 \\
\text { cpm. }\end{array}$ & $\begin{array}{ll}\text { BMI, WC, } & \text { SBP, DBP } \\
\text { and } & \text { Non-HDL- } \\
\text { Cholesterol. }\end{array}$ & $\begin{array}{l}\text { Linear regression analyses } \\
\text { Confounders: Age, average } \\
\text { daily minutes of MVPA on } \\
\text { valid days and } \\
\text { accelerometer wear time. }\end{array}$ & $\begin{array}{l}\text { Number of breaks in sedentary } \\
\text { time, after } 3 \text { pm on weekdays, was } \\
\text { negatively associated with WC } \\
(\beta=-4.04, p<0.006) \text { in boys aged } \\
11-14 \text { years. } \\
\text { Breaks in sedentary time were not } \\
\text { significantly associated with BMI } \\
\text { or WC in girls of any age or in boys } \\
\text { aged } 6-10 \text { or } 15-19 \text { years. } \\
\text { Breaks in sedentary time were not } \\
\text { associated with BP or non-HDL } \\
\text { cholesterol in boys or girls. }\end{array}$ \\
\hline $\begin{array}{l}\text { Saunders et al., } \\
2013 \\
\text { (QUebec } \\
\text { Adiposity and } \\
\text { Lifestyle } \\
\text { InvesTigation } \\
\text { in Youth - } \\
\text { QUALITY - } \\
\text { cohort) [19] }\end{array}$ & $\begin{array}{c}\text { Cross- } \\
\text { sectional }\end{array}$ & $\begin{array}{c}\text { Canada: } 522 ; \\
\text { mean age }=9.2 \\
(9.0-9.3) \text { years } \\
(45 \% \text { girls })\end{array}$ & $\begin{array}{l}\text { Actigraph LS } \\
7164 \\
\text { accelerometer } \\
\text { (uniaxial) }\end{array}$ & $\begin{array}{l}\text { Breaks in sedentary time } \\
\text { were defined as any } \\
\text { interruption in } \\
\text { sedentary time lasting } \\
\text { one minute or longer, in } \\
\text { which the accelerometer } \\
\text { cpm, rose up to or above } \\
100 .\end{array}$ & $\begin{array}{l}\text { BMI } \quad z \text {-score and } \\
\text { continuous } \\
\text { cardiometabolic risk } \\
\text { score } \quad(-z \mathrm{HDL}+ \\
\text { zInsulin+ zGlucose+ } \\
\text { zTriglycerides+ } \\
(z \mathrm{BMI}+z \mathrm{WC}) / 2+ \\
(z \mathrm{SBP}+z \mathrm{DBP}) / 2)\end{array}$ & $\begin{array}{l}\text { Linear regression analyses } \\
\text { Confounders: } \\
\text { Accelerometer wear time, } \\
\text { age, light PA, MVPA, total } \\
\text { sedentary time, BMI Z- } \\
\text { score (unless included in } \\
\text { the outcome), Tanner } \\
\text { stage, parental income and } \\
\text { level of education. }\end{array}$ & $\begin{array}{l}\text { Breaks in sedentary time were } \\
\text { negatively associated with the } \\
\text { continuous cardiometabolic risk } \\
\text { score, both in boys and girls (boys: } \\
\beta=-0.057,95 \% \mathrm{CI}=-0.106 \text { to } \\
0.008 \text {; girls: } \beta=-0.084,95 \% \mathrm{CI}=- \\
0.143 \text { to }-0.024 \text { ) and } \mathrm{BMI} z \text {-scores } \\
\text { (boys: } \beta=-0.026,95 \% \mathrm{CI}=-0.040 \text { to } \\
-0.012 \text {; girls: } \beta=-0.032,95 \% \mathrm{CI}=- \\
0.048 \text { to }-0.016 \text { ). All } p<0.05 \text {. }\end{array}$ \\
\hline $\begin{array}{l}\text { Carson et al., } \\
2014 \\
\text { Project BEAT }\end{array}$ & $\begin{array}{c}\text { Cross- } \\
\text { sectional }\end{array}$ & $\begin{array}{c}\text { Canada: } 787 ; \\
\text { mean age }= \\
11.1 \pm 0.7 \text { years }\end{array}$ & $\begin{array}{l}\text { ActiGraph GT1M } \\
\text { accelerometer } \\
\text { (uniaxial) }\end{array}$ & $\begin{array}{l}\text { A break was defined as } \\
\text { an interruption in } \\
\text { sedentary time and }\end{array}$ & $\begin{array}{l}\text { BMI } z \text {-score and BMI } \\
z \text {-score stratified by } \\
\text { low and high MVPA. }\end{array}$ & $\begin{array}{l}\text { Multiple linear regression } \\
\text { models }\end{array}$ & $\begin{array}{l}\text { After adjustment for confounders, } \\
\text { the total number of sedentary } \\
\text { breaks per hour, the total number }\end{array}$ \\
\hline
\end{tabular}


Sousa-Sá E et al.,/2020

\begin{tabular}{|c|c|c|c|c|c|c|c|}
\hline $\begin{array}{l}\text { (built } \\
\text { environment } \\
\text { and active } \\
\text { transport) [21] }\end{array}$ & & (54.3\% girls) & & $\begin{array}{l}\text { reflected any } 5 \text {-s epoch } \\
\text { change from sedentary } \\
\text { time to light-intensity PA } \\
\text { or MVPA. }\end{array}$ & & $\begin{array}{l}\text { Confounders: Age, sex, SES, } \\
\text { MVPA and total sedentary } \\
\text { time. }\end{array}$ & $\begin{array}{l}\text { of sedentary breaks per hour on } \\
\text { weekdays and on weekends were } \\
\text { not associated with BMI } z \text {-score } \\
\text { nor BMI } z \text {-score when participants } \\
\text { were stratified by low and high } \\
\text { MVPA ( } p>0.005 \text { for all). }\end{array}$ \\
\hline $\begin{array}{l}\text { Dowd et al., } \\
2014 \text { [31] }\end{array}$ & $\begin{array}{c}\text { Cross- } \\
\text { sectional }\end{array}$ & $\begin{array}{l}\text { Ireland: } 195 ; \\
\text { mean } \\
\text { age }=15.7 \pm 0.9 \\
\text { years } \\
(100 \% \text { girls })\end{array}$ & $\begin{array}{c}\text { ActivPAL } \\
\text { inclinometer }\end{array}$ & $\begin{array}{l}\text { Breaks in sitting time } \\
\text { were defined as any } \\
\text { transition from a } \\
\text { sitting/lying posture to a } \\
\text { standing posture and } \\
\text { breaks in sitting/lying } \\
\text { time were summed over } \\
\text { the waking } \\
\text { measurement period. }\end{array}$ & $\begin{array}{l}\text { BMI and skinfolds } \\
\text { (biceps, } \\
\text { subscapular and iliac } \\
\text { crest). }\end{array}$ & $\begin{array}{l}\text { Linear mixed-model } \\
\text { Confounders: Age and the } \\
\text { clustering of participants } \\
\text { within schools. }\end{array}$ & $\begin{array}{l}\text { A significant association was } \\
\text { observed between the number of } \\
\text { breaks in sitting/lying time and } \\
\text { BMI percentiles }(\beta=-0.3 ; 95 \% \text { CI - } \\
0.59 \text { to }-0.01 ; p=0.04) \text {, but not } \\
\text { skinfolds }(\beta=-0.3 ; 95 \% \mathrm{CI}-0.63 \text { to } \\
0.03 ; p=0.07) \text {, after adjustments. }\end{array}$ \\
\hline $\begin{array}{l}\text { Gabel et al., } \\
2016 \\
\text { (The } \\
\text { Transform-Us! } \\
\text { Study) [23] }\end{array}$ & $\begin{array}{c}\text { Cross- } \\
\text { sectional }\end{array}$ & $\begin{array}{l}\text { Australia: } 164 ; \\
\text { mean } \\
\text { age }=8.7 \pm 0.4 \\
\text { years } \\
\text { (53\% girls) }\end{array}$ & $\begin{array}{l}\text { ActiGraph GT3X } \\
\text { accelerometer } \\
\text { (triaxial) }\end{array}$ & $\begin{array}{l}\text { Breaks in sedentary time } \\
\text { were defined as the } \\
\text { number of times that the } \\
\text { accelerometer exceeded } \\
25 \text { counts per 15-s } \\
\text { epoch, following a } 15-\mathrm{s} \\
\text { epoch of }<25 \text { counts per } \\
\text { epoch. }\end{array}$ & $\begin{array}{l}\text { WC and C-reactive } \\
\text { protein. }\end{array}$ & $\begin{array}{l}\text { General linear models } \\
\text { Confounders: Clustering } \\
\text { within school classes, } \\
\text { child's sex and WC, } \\
\text { accelerometry-derived } \\
\text { MVPA and diet density. }\end{array}$ & $\begin{array}{l}\text { No significant associations } \\
\text { between the frequency of breaks in } \\
\text { sedentary time and C-reactive } \\
\text { protein were observed, } \\
\text { independent of confounders. }\end{array}$ \\
\hline $\begin{array}{l}\text { Bailey et al., } \\
2017 \\
\text { (The HAPPY } \\
\text { study) [30] }\end{array}$ & $\begin{array}{c}\text { Cross- } \\
\text { sectional }\end{array}$ & $\begin{array}{c}\text { UK: } 111 ; \text { mean } \\
\text { age }=11.8 \pm 1.4 \\
\text { years } \\
\text { (59\% girls) }\end{array}$ & $\begin{array}{c}\text { RT3 } \\
\text { accelerometer } \\
\text { (triaxial) }\end{array}$ & $\begin{array}{l}\text { Breaks in sedentary time } \\
\text { were defined as a } \\
\text { sedentary bout } \\
\text { interrupted by } \geq 1 \text { min of } \\
\text { light activity or MVPA. }\end{array}$ & 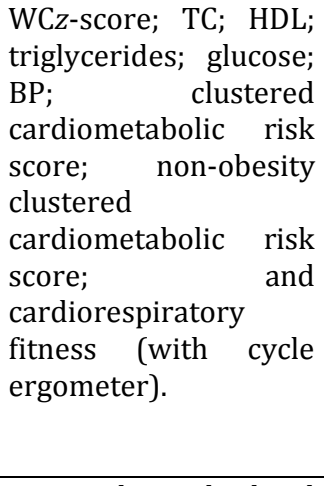 & $\begin{array}{l}\text { Multiple logistic regression } \\
\text { Confounders: } \quad \text { Sex, } \\
\text { ethnicity, total sedentary } \\
\text { time, MVPA, accelerometer } \\
\text { wear time and light PA. }\end{array}$ & 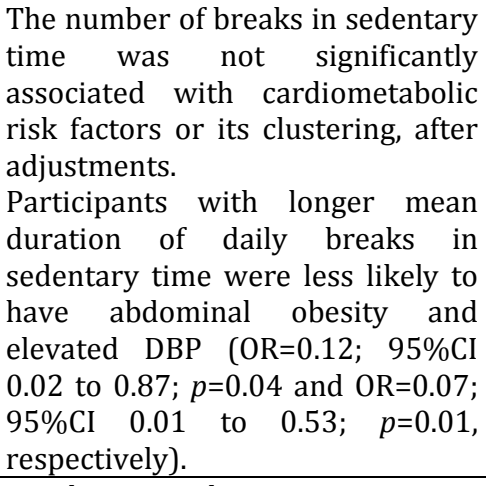 \\
\hline $\begin{array}{l}\text { Júdice et al., } \\
2017 \text { [32] }\end{array}$ & $\begin{array}{c}\text { Cross- } \\
\text { sectional }\end{array}$ & $\begin{array}{c}\text { Portugal: } 2698 ; \\
\text { mean } \\
\text { age }=13.4 \pm 2.28 \\
\text { years } \\
\text { (53.2\% girls) }\end{array}$ & $\begin{array}{l}\text { ActiGraph, GT1M } \\
\text { accelerometer } \\
\text { (uniaxial) }\end{array}$ & $\begin{array}{l}\text { Breaks in sedentary time } \\
\text { were defined as each } \\
\text { interruption in } \\
\text { sedentary time in which } \\
\text { the accelerometer count } \\
\text { raised up to or above } \\
100 \text { counts/min and } \\
\text { which stayed within the }\end{array}$ & $\begin{array}{l}\text { BMI and standardized } \\
\text { fitness composite } \\
\text { score }(z \text {-score }) .\end{array}$ & $\begin{array}{l}\text { Linear regression models } \\
\text { Confounders: Age, season } \\
\text { of data collection, total } \\
\text { sedentary time and MVPA. }\end{array}$ & $\begin{array}{l}\text { Breaks in sedentary time were } \\
\text { positively associated with fitness } \\
\text { after adjustments, independent of } \\
\text { total sedentary time and MVPA in } \\
\text { boys but not in girls (all: } \beta=0.024 \text {, } \\
95 \% \text { CI } 0.013 \text { to } 0.034 ; \text { boys: } \\
\beta=0.026,95 \% \text { CI } 0.009 \text { to } 0.042 \text {; } \\
\text { girls: } \beta=0.001,95 \% \text { CI }-0.017 \text { to }\end{array}$ \\
\hline
\end{tabular}


Sousa-Sá E et al.,/2020

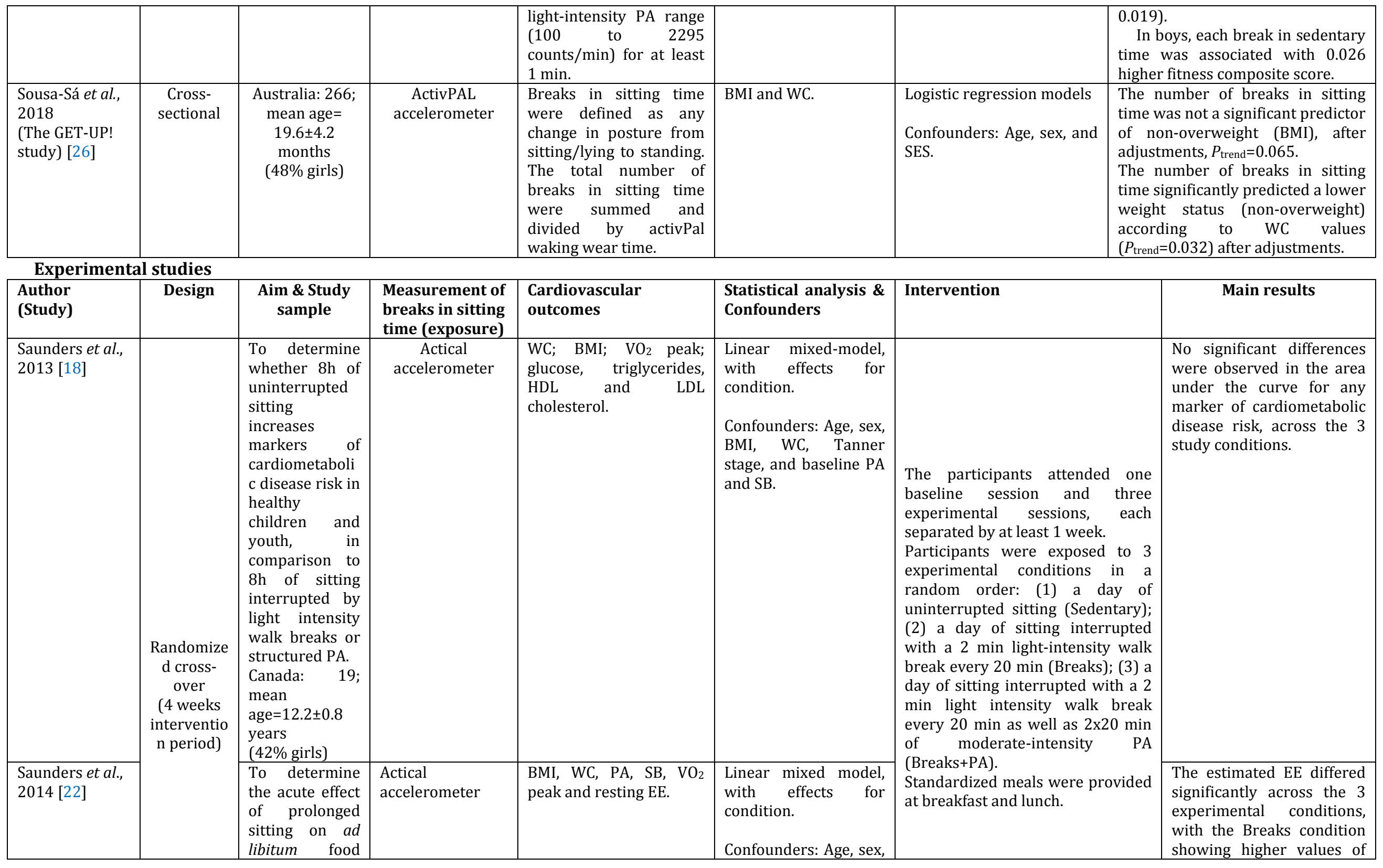


Sousa-Sá E et al.,/2020

\begin{tabular}{|c|c|c|c|c|c|c|c|}
\hline & & $\begin{array}{l}\text { intake and } \\
\text { spontaneous } \\
\text { PA levels in } \\
\text { healthy } \\
\text { children and } \\
\text { youth. } \\
\text { Canada: } 20 \text {; } \\
\text { mean } \\
\text { age }=12.2 \pm 0.9 \\
\text { years } \\
(40 \% \text { girls })\end{array}$ & & & $\begin{array}{l}\text { Tanner stage, BMI, } \\
\text { baseline PA and SB } \\
\text { and accelerometer } \\
\text { wear time. }\end{array}$ & & $\begin{array}{l}\text { EE than the Sedentary } \\
\text { condition and the } \\
\text { Breaks+PA condition } \\
\text { showing higher values of } \\
\text { EE than the Breaks } \\
\text { condition (all } p<0.01 \text { ). }\end{array}$ \\
\hline $\begin{array}{l}\text { Belcher et al., } \\
2015 \text { [28] }\end{array}$ & $\begin{array}{l}\text { Randomize } \\
\text { d trial } \\
(2 \text { months })\end{array}$ & $\begin{array}{l}\text { To investigate } \\
\text { whether } \\
\text { interrupting } \\
\text { sitting with } \\
\text { short, } \\
\text { moderate- } \\
\text { intensity } \\
\text { walking bouts } \\
\text { improved } \\
\text { glucose } \\
\text { tolerance in } \\
\text { children. } \\
\text { USA: } 28 ; \text { mean } \\
\text { age=10.2 } 1.5 \\
\text { years } \\
\text { (46\% girls) }\end{array}$ & $\begin{array}{l}\text { Actigraph GT3X+ } \\
\text { (triaxial } \\
\text { accelerometer) }\end{array}$ & $\begin{array}{l}\text { Insulin, } \\
\text { glucose and free fatty } \\
\text { acids }\end{array}$ & $\begin{array}{l}\text { ANOVA } \\
\text { Confounders: } \\
\text { Randomization order }\end{array}$ & $\begin{array}{l}\text { Participants underwent two } \\
\text { experimental conditions in random } \\
\text { order on different days: } \\
\text { continuous sitting for } 3 \text { hours or } \\
\text { sitting interrupted by walking ( } 3 \\
\text { min of moderate-intensity walking } \\
\text { every 30min). }\end{array}$ & $\begin{array}{l}\text { Interrupting sitting } \\
\text { resulted in a } 32 \% \text { lower } \\
\text { insulin AUC }(p<0.001) \text {, } \\
17 \% \text { lower C-peptide AUC } \\
(p<0.001) \text {, and } 7 \% \text { lower } \\
\text { glucose AUC }(p=0.018) \text { vs } \\
\text { continuous sitting. Mixed } \\
\text { model results indicated } \\
\text { that insulin }(p=0.036) \text { and } \\
\text { free fatty acid } \\
\text { concentrations (p=0.009) } \\
\text { were significantly lower in } \\
\text { the interrupted vs the } \\
\text { continuous sitting } \\
\text { condition. }\end{array}$ \\
\hline $\begin{array}{l}\text { Contardo-Ayala } \\
\text { et al., 2016 } \\
\text { (Make A Stand } \\
\text { Kids Study - } \\
\text { MASK) [24] }\end{array}$ & $\begin{array}{l}\text { Pilot non- } \\
\text { randomize } \\
\mathrm{d} \text { trial } \\
\text { (8-months } \\
\text { interventio } \\
\mathrm{n} \text { period) }\end{array}$ & $\begin{array}{l}\text { To examine the } \\
\text { impact of an 8- } \\
\text { month } \\
\text { intervention } \\
\text { incorporating } \\
\text { height- } \\
\text { adjustable } \\
\text { desks and } \\
\text { pedagogical } \\
\text { strategies on } \\
\text { overall volume } \\
\text { and pattern of } \\
\text { sitting time in } \\
\text { the classroom, } \\
\text { cardio- }\end{array}$ & $\begin{array}{l}\text { ActivPAL } \\
\text { inclinometer }\end{array}$ & $\begin{array}{l}\text { WC } z \text {-score; BMI } z \text {-score } \\
\text { and BP. }\end{array}$ & $\begin{array}{l}\text { Hierarchical linear } \\
\text { mixed models. } \\
\text { Confounders: Baseline } \\
\text { values. }\end{array}$ & $\begin{array}{l}\text { One Year } 6 \text { class was assigned as } \\
\text { an IG and another Year } 6 \text { class as a } \\
\text { CG. Each participant in the IG was } \\
\text { provided with an adjustable } \\
\text { height-adjustable workstation. The } \\
\text { pedagogical strategies } \\
\text { recommended that extended } \\
\text { classroom teaching blocks (of at } \\
\text { least } 60 \text { min) would be interrupted } \\
\text { every } 30 \text { min with a 2-min guided } \\
\text { light-intensity active break from } \\
\text { sitting. The teacher was asked to } \\
\text { deliver at least one } 30 \text {-min } \\
\text { standing lesson each day. } \\
\text { The CG followed standard }\end{array}$ & $\begin{array}{l}\text { During school time, the IG } \\
\text { exhibited a significant } \\
\text { positive mean difference in } \\
\text { the frequency of sit to } \\
\text { stand transitions relative to } \\
\text { the CG (mean dif }=7.26 \text {; } \\
95 \% \text { CI } 1.2 \text { to 13.32; } \\
p=0.02 \text { ). No other } \\
\text { significant intervention } \\
\text { effects were reported for } \\
\text { other sitting and activity } \\
\text { measures, during } \\
\text { classroom time, on waking } \\
\text { hours. } \\
\text { No significant intervention }\end{array}$ \\
\hline
\end{tabular}


Sousa-Sá E et al.,/2020

\begin{tabular}{|c|c|c|c|c|c|c|c|}
\hline & & $\begin{array}{l}\text { metabolic risk } \\
\text { factors and } \\
\text { perceptions of } \\
\text { musculoskeleta } \\
\text { l discomfort } \\
\text { among primary } \\
\text { school children. } \\
\text { Australia: 48; } \\
\text { mean } \\
\text { age }=11.6 \pm 0.3 \\
\text { years } \\
\text { (58\% girls) }\end{array}$ & & & & $\begin{array}{l}\text { pedagogical practice and retained } \\
\text { traditional classroom furniture. }\end{array}$ & $\begin{array}{l}\text { effects were found for BMI, } \\
\text { WC or BP. }\end{array}$ \\
\hline $\begin{array}{l}\text { Broadney et al., } \\
2018 \text { [29] }\end{array}$ & $\begin{array}{c}\text { Randomize } \\
\mathrm{d} \\
\text { crossover } \\
\text { trial } \\
(1 \text { month) }\end{array}$ & 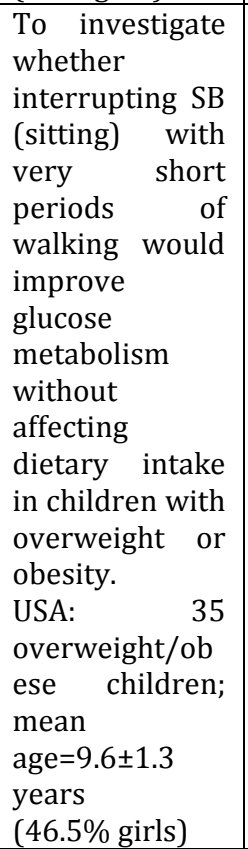 & $\begin{array}{l}\text { Actigraph GT3X+ } \\
\text { wrist } \\
\text { accelerometer }\end{array}$ & $\begin{array}{l}\text { Insulin, C-peptide, and } \\
\text { glucose. }\end{array}$ & 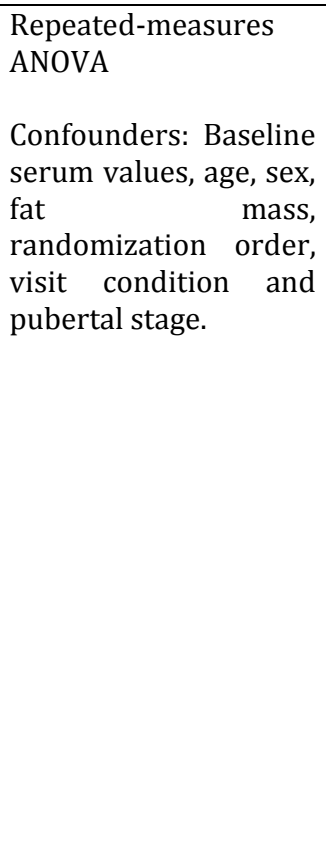 & $\begin{array}{l}\text { Participants underwent } \\
\text { experimental conditions in random } \\
\text { order: prolonged sitting (3h of } \\
\text { continuous sitting) } \quad \text { and } \\
\text { interrupted sitting (3min of } \\
\text { moderate-intensity walking at } \\
\text { 80\% of ventilatory threshold every } \\
30 \text { min for } 3 \mathrm{~h}) \text {. }\end{array}$ & $\begin{array}{l}\text { Results identified lower } \\
\text { insulin and C-peptide in the } \\
\text { interrupted condition } \\
(p=0.007 \text { and } p=0.029, \\
\text { respectively). } \\
\text { The intervention reduced } \\
\text { insulin AUC by } 21 \% \\
(p<0.001) \text { and C-peptide } \\
\text { AUC 18\% ( } p=0.001) \text { and } \\
\text { improved estimated insulin } \\
\text { sensitivity }(p=0.013) \text {. }\end{array}$ \\
\hline $\begin{array}{l}\text { Contardo-Ayala } \\
\text { et al., } 2018 \text { [25] }\end{array}$ & $\begin{array}{l}\text { Quasi- } \\
\text { experimen } \\
\quad \text { tal } \\
\text { interventio } \\
\mathrm{n} \text { trial } \\
\text { (17 weeks } \\
\text { interventio } \\
\mathrm{n} \text { period) }\end{array}$ & $\begin{array}{l}\text { To investigate } \\
\text { the impact of } \\
\text { an intervention } \\
\text { to reduce } \\
\text { classroom } \\
\text { sitting time on } \\
\text { adolescents' EE } \\
\text { (kcal/lesson), } \\
\text { BMI, WC and }\end{array}$ & $\mathrm{N} / \mathrm{A}$ & EE, BMI and WC. & $\begin{array}{lr}\text { Hierarchical } & \text { linear } \\
\text { mixed models. } & \\
\text { Confounders: } \\
\begin{array}{l}\text { Clustering } \\
\text { (intervention }\end{array} \text { offect } \\
\begin{array}{l}\text { comparison groups), } \\
\text { baseline values, age } \\
\text { and sex. }\end{array}\end{array}$ & $\begin{array}{l}\text { Students from years } 7,10 \text { and } 11 \\
\text { with timetabled classes in the IG } \\
\text { and a CG of students (i.e. matched } \\
\text { on year level and school subject) } \\
\text { who only used the traditional } \\
\text { 'seated' classrooms. Traditional } \\
\text { classroom furniture in the IG was } \\
\text { replaced with height-adjustable } \\
\text { desks and lab stools for every }\end{array}$ & $\begin{array}{l}\text { Compared to the CG, the IG } \\
\text { expended significantly } \\
\text { more energy during a } \\
\text { typical lesson at } 4 \text { and } 17 \\
\text { weeks. Relative to the CG, } \\
\text { WC was significantly lower } \\
\text { in the IG at } 4 \text { and } 17 \text { weeks. } \\
\text { While BMI was significantly } \\
\text { higher at } 4 \text { weeks, no }\end{array}$ \\
\hline
\end{tabular}


Sousa-Sá E et al.,/2020

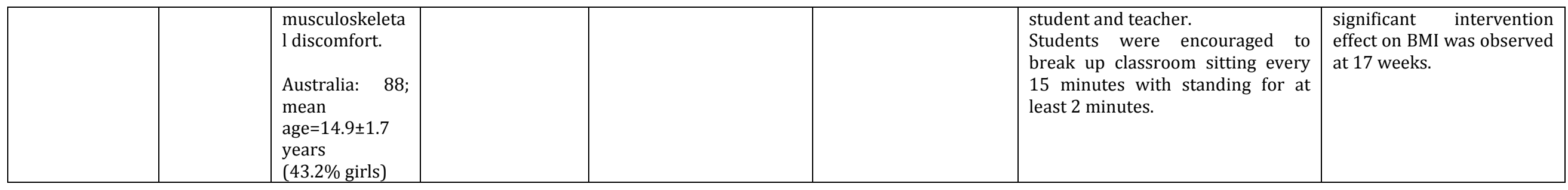

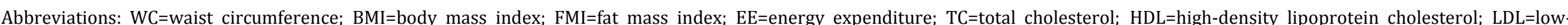

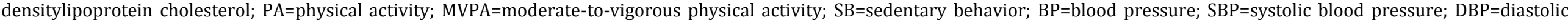
blood pressure; $\mathrm{CG}=$ control group; $\mathrm{IG}=$ intervention group; $\mathrm{AUC}=$ area under the curve; $\mathrm{cpm}=$ counts per minute; $\mathrm{SF}=$ fragmentation of sedentary time; $\mathrm{SES}=$ socio economic status.

Table 2. Risk of bias for included studies

\begin{tabular}{|c|c|c|c|c|c|c|c|c|c|c|}
\hline \multirow{3}{*}{ STUDY } & \multicolumn{2}{|c|}{ 1.SELECTION BIAS } & 2.ATTRITION BIAS & \multicolumn{2}{|c|}{ 3.DETECTION BIAS } & \multicolumn{2}{|c|}{ 4.PERFORMANCE BIAS } & $\begin{array}{c}\text { 5.SELECTIVE } \\
\text { REPORTING } \\
\text { BIAS }\end{array}$ & & \\
\hline & $\begin{array}{l}\text { Clear } \\
\text { criteria } \\
\text { for those } \\
\text { included } \\
\text { and/or } \\
\text { excluded? }\end{array}$ & $\begin{array}{l}\text { Was the } \\
\text { sample } \\
\text { randomly } \\
\text { selected? }\end{array}$ & $\begin{array}{l}\text { Did an adequate } \\
\text { proportion (at least } 70 \% \text { ) } \\
\text { of those consenting to } \\
\text { participate in the study } \\
\text { have complete data? } \\
\text { (Incomplete follow-up; } \\
\text { high loss to follow-up; } \\
\text { missing data) }\end{array}$ & $\begin{array}{l}\text { Did the study } \\
\text { report the sources } \\
\text { and details of the } \\
\text { tool used in the } \\
\text { study to assess } \\
\text { cardiovascular } \\
\text { health? }\end{array}$ & $\begin{array}{l}\text { Was the tool } \\
\text { used in the } \\
\text { study to assess } \\
\text { cardiovascular } \\
\text { health reliable } \\
\text { and/or valid? }\end{array}$ & $\begin{array}{l}\text { Did the study report } \\
\text { the sources and details } \\
\text { of the measurement } \\
\text { tool used in the study } \\
\text { for the exposures? } \\
\text { (Exposures: measures } \\
\text { of breaks in sitting } \\
\text { time) }\end{array}$ & $\begin{array}{l}\text { Were the } \\
\text { measurements } \\
\text { of exposure in } \\
\text { this study } \\
\text { reliable } \\
\text { and/or valid? }\end{array}$ & $\begin{array}{l}\text { Did the study } \\
\text { have complete } \\
\text { data and/or } \\
\text { reports all } \\
\text { outcomes and } \\
\text { not others } \\
\text { based on the } \\
\text { results? }\end{array}$ & & \\
\hline & Rating & Rating & Rating & Rating & Rating & Rating & Rating & Rating & Score* & \\
\hline $\begin{array}{l}\text { Carson \& } \\
\text { Janssen, } 2011 \\
{[27]}\end{array}$ & 0 & 1 & 0 & 1 & 1 & 1 & 1 & 1 & $6 / 8$ & Low \\
\hline $\begin{array}{l}\text { Colley et al., } \\
2013 \text { [20] }\end{array}$ & 0 & 1 & 1 & 1 & 1 & 1 & 1 & 1 & $7 / 8$ & Low \\
\hline $\begin{array}{l}\text { Saunders et al., } \\
2013[18]\end{array}$ & 0 & 0 & 1 & 1 & 1 & 1 & 1 & 1 & $5 / 8$ & High \\
\hline $\begin{array}{l}\text { Saunders et al., } \\
2013 \text { [19] }\end{array}$ & 1 & 0 & 1 & 1 & 1 & 1 & 1 & 1 & $7 / 8$ & Low \\
\hline Carson et al., & 0 & 0 & 1 & 1 & 1 & 1 & 1 & 1 & $6 / 8$ & Low \\
\hline
\end{tabular}


Sousa-Sá E et al.,/2020

\begin{tabular}{|c|c|c|c|c|c|c|c|c|c|c|}
\hline 2014 [21] & & & & & & & & & & \\
\hline $\begin{array}{l}\text { Dowd et al., } \\
2014 \text { [31] }\end{array}$ & 1 & 1 & 1 & 1 & 1 & 1 & 1 & 1 & $8 / 8$ & Low \\
\hline $\begin{array}{l}\text { Saunders et al., } \\
2014 \text { [22] }\end{array}$ & 0 & 0 & 0 & 1 & 1 & 1 & 1 & 1 & $5 / 8$ & High \\
\hline $\begin{array}{l}\text { Belcher et al., } \\
2015 \text { [28] }\end{array}$ & 1 & 1 & 0 & 1 & 1 & 1 & 1 & 1 & $7 / 8$ & Low \\
\hline $\begin{array}{l}\text { Contardo- } \\
\text { Ayala et al., } \\
2016 \text { [24] }\end{array}$ & 0 & 0 & 0 & 1 & 1 & 1 & 1 & 1 & $5 / 8$ & High \\
\hline $\begin{array}{l}\text { Gabel et al., } \\
2016 \text { [23] }\end{array}$ & 0 & 0 & 0 & 1 & 1 & 1 & 1 & 1 & $5 / 8$ & High \\
\hline $\begin{array}{l}\text { Bailey et al., } \\
2017 \text { [30] }\end{array}$ & 1 & 0 & 0 & 1 & 1 & 1 & 1 & 1 & $6 / 8$ & Low \\
\hline $\begin{array}{l}\text { Júdice et al., } \\
2017 \text { [32] }\end{array}$ & 1 & 1 & 1 & 1 & 1 & 1 & 1 & 1 & $8 / 8$ & Low \\
\hline $\begin{array}{l}\text { Broadney et } \\
\text { al., } 2018 \text { [29] }\end{array}$ & 1 & 1 & 0 & 1 & 1 & 1 & 1 & 1 & $7 / 8$ & Low \\
\hline $\begin{array}{l}\text { Contardo- } \\
\text { Ayala et al., } \\
2018 \text { [25] }\end{array}$ & 0 & 0 & 0 & 1 & 1 & 1 & 1 & 1 & $5 / 8$ & High \\
\hline $\begin{array}{l}\text { Sousa-Sá et al., } \\
2018 \text { [26] }\end{array}$ & 1 & 1 & 1 & 1 & 1 & 1 & 1 & 1 & $8 / 8$ & Low \\
\hline & $7 / 15$ & $6 / 15$ & $7 / 15$ & $15 / 15$ & $15 / 15$ & $15 / 15$ & $15 / 15$ & $15 / 15$ & & \\
\hline
\end{tabular}

$0=$ No; $1=$ Yes;

* Scores above $6 / 8$ were considered low risk of bias. 
Sousa-Sá E et al.,/2020

Table 3. Results of the included studies

\begin{tabular}{|c|c|c|c|c|}
\hline & & Favorable associations & Null associations & Summary \\
\hline \multirow{4}{*}{ 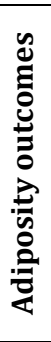 } & BMI & Dowd et al., 2014 [31] & $\begin{array}{l}\text { Colley et al., } 2013[20] \\
\text { Sousa-Sá et al., } 2018[26] \\
\text { Contardo-Ayala et al., 2016 [24]* } \\
\text { Contardo-Ayala et al., } 2018[25]^{*}\end{array}$ & \multirow[t]{4}{*}{$\begin{array}{l}\text { 5/7 studies showed favorable associations for adiposity outcomes } \\
5 / 7 \text { studies showed null associations for adiposity outcomes }\end{array}$} \\
\hline & zBMI & Saunders et al., 2013 [19] & Carson et al., 2014 [21] & \\
\hline & WC & $\begin{array}{l}\text { Colley et al., } 2013[20] \\
\text { Sousa-Sá et al., } 2018[26] \\
\text { Contardo-Ayala et al., } 2018[25]^{*}\end{array}$ & $\begin{array}{l}\text { Colley et al., } 2013 \text { [20] } \\
\text { Contardo-Ayala et al., } 2016 \text { [24]* }\end{array}$ & \\
\hline & Skinfolds & & Dowd et al., 2014 [31] & \\
\hline & & & & \\
\hline \multirow{12}{*}{ 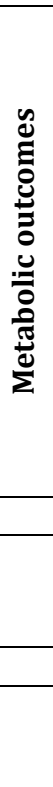 } & Cholesterol & & Colley et al., 2013 [20] & \multirow[t]{8}{*}{ 3/9 studies showed favorable associations for metabolic outcomes } \\
\hline & C-reactive protein & & Gabel et al., 2016 [23] & \\
\hline & Insulin & $\begin{array}{l}\text { Belcher et al., } 2015[28]^{*} \\
\text { Broadney et al., } 2018 \text { [29]* }\end{array}$ & & \\
\hline & C-peptide & $\begin{array}{l}\text { Belcher et al., } 2015[28]^{*} \\
\text { Broadney et al., } 2018[29]^{*}\end{array}$ & & \\
\hline & Glucose & Belcher et al., 2015 [28]* & & \\
\hline & Free fatty acid & Belcher et al., 2015 [28]* & & \\
\hline & BP & & $\begin{array}{l}\text { Colley et al., } 2013 \text { [20] } \\
\text { Contardo-Ayala et al., } 2016[24]^{*}\end{array}$ & \\
\hline & Cardio metabolic risk & Saunders et al., 2013 [19] & $\begin{array}{l}\text { Carson et al., } 2011[27] \\
\text { Bailey et al., } 2017 \text { [30] } \\
\text { Saunders et al., } 2013[18]^{*}\end{array}$ & \\
\hline & & & & \\
\hline & Fitness & Júdice et al., 2017 [32] & & $\begin{array}{l}\text { 1/1 studies showed favorable associations for fitness measures } \\
0 \text { studies showed favorable associations for fitness measures }\end{array}$ \\
\hline & & & & \\
\hline & $\mathbf{E E}$ & $\begin{array}{l}\text { Saunders et al., } 2014 \text { [22]* } \\
\text { Contardo-Ayala et al., } 2018 \text { [25]* }\end{array}$ & & $\begin{array}{l}\text { 2/2 studies showed favorable associations for energy expenditure } \\
0 \text { studies showed favorable associations for energy expenditure }\end{array}$ \\
\hline
\end{tabular}

Abbreviations: BMI=body mass index; WC=waist circumference; FMI=fat mass index; EE=energy expenditure

References with an $\left({ }^{*}\right)$ are experimental studies; references without an $(*)$ are observational studies. 


\section{Sousa-Sá E et al.,/2020}

\section{Results}

A total of 3262 studies, published between 1937 and 2018, were identified through database searches (Figure 1). After removing duplicates, 2577 studies remained. After titles and abstracts were screened, 315 full-text articles were included for full-text review and, of these, 15 studies met the inclusion criteria.

Included studies were published between 2011 and 2018 and were conducted in Canada [1822], Australia [23-26], United States [27-29], UK [30], Ireland [31] and Portugal [32]. Sample size ranged between 19 and 2698 children and adolescents (43\% girls). This review included a total of 9116 participants. Nine studies were crosssectional in design (60\%), three were randomized crossover trials $(20 \%)$, and there was one randomized trial $(6.7 \%)$, one pilot non-randomized trial (6.7\%) and one quasi-experimental intervention trial (6.6\%). Samples age ranged from 1.3 to 16.6 years. Sedentary time was measured with Actical [18, 22, 20], Actigraph accelerometers (AM7124; LS7164; GT1M; GT3X; GT3X+)[27, 21, 23, $32,29,19,28]$, activPal accelerometer $[24,26]$ and RT3 triaxial accelerometer [30]. Of the 15 included studies, only five (33\%) showed consistent and significant associations. Results from the observational studies' showed that breaks in sedentary time were negatively associated with the continuous cardiometabolic risk score and with BMI $z$-scores [19]; and breaks in sedentary time were positively associated with fitness [32]. Experimental studies' showed that the higher the number of breaks, the higher the energy expenditure [22]; and interrupting sitting time resulted in lower insulin, Cpeptide, glucose and lower free fatty acids $[29,28]$. The remaining 10 studies (67\%), showed either favorable or null associations between breaks in sedentary time/sitting time and cardiovascular health $[27,20,21,31,23,30,26,18,19,25]$. Results also showed a similar number of favorable and null associations for both adiposity and metabolic outcomes; whereas for fitness and energy expenditure, results only showed favorable associations (Table 3).
Out of the fifteen studies included in this review, ten studies (67\%) were classified as having a low ROB and five as having a high ROB (33\%). All studies had a reliable and/or valid tool to assess both cardiovascular health outcomes and breaks in sitting time.

\section{Discussion}

Research on sedentary behavior has typically focused on the investigation of its volumes and types. However, the patterns in which sedentary behavior is accumulated should also be taken into consideration given that the way in which sedentary time is accumulated may help to clarify the associations between sedentary time and health outcomes, in children and adolescents [28].

Most of the studies included in this review reported favorable and some null associations between breaking sitting time and CV health outcomes. This may be due to the use of several operational definitions for sedentary breaks in sedentary time/sitting time, as well as different devices used to assess breaks in sitting time. Indeed, only recently, standardized definitions of sitting time and breaks in sitting time have been proposed [1]. The different definitions of breaks used across studies is one of the reasons for the different number of breaks in all studies; i.e. Dowd and colleagues [31] defined breaks in sitting time as any transition from a sitting/lying posture to a standing posture, whereas Gabel [23] and colleagues defined breaks in sedentary time as the number of times that the accelerometer exceeded 25 counts per 15 -s epoch, following a 15-s epoch of $<25$ counts per epoch. Within those definitions, the authors also added the concept of "breakers" and "prolongers", to distinguish those who accumulate sitting time with frequent interruptions from those who accumulate sitting time in prolonged and continuous periods, respectively. Such definitions have the potential to be used in future studies. This would significantly reduce the heterogeneity between studies, making comparisons between studies easier and attainable. Moreover, null associations were seen in studies with adequate sample power $[33,20]$, i.e. samples 


\section{Sousa-Sá E et al.,/2020}

big enough to address the research question, as well as studies with small samples [24, 25]. Therefore, we cannot assume that sample size was a predominate factor influencing the results.

The included studies assessed breaks in sitting time with different devices, as stated before. In this regards it is worthy to comment that only the most recent accelerometers models (GT3X and GT3X+) are able to differentiate between various postures (i.g. lying, sitting, standing) [33, 34]; whereas the activPal allows for this [35]. As a consequence of potential misclassification when sedentary/sitting time are measured by accelerometry, time spent sedentary (both total and breaks) may be overestimated [36]. Nevertheless, we considered that all studies used valid tools to assess breaks in sitting time, given the definitions of breaks that each of the included studies considered. Regardless of the inconsistencies found, the results of our systematic review offer new data regarding the associations of breaking up prolonged time spent sitting, with either LPA or MVPA, on the children's cardiometabolic profile.

While increased breaks in objectively measured sedentary time have been positively associated with markers of cardiometabolic risk in adults $[11,12]$, this is not yet a certainty in the pediatric population. However, in the absence of more longitudinal and epidemiological evidence, we can only speculate that the harmful effects of prolonged sitting time in youngsters, take time to exert an effect on health. This could potentially be a possible explanation for the different results between children/adolescents and adult population. However, and remarkably, the UK Physical Activity Guidelines, and the new 24-hour Movement Australian and Canadian Guidelines for the Early Years [7, 37, 8] recommend on reducing/breaking up sedentary behavior, more precisely, sitting time. Both the Australian and Canadian guidelines, further recommend that sitting time should be interrupted, at least, every hour, and it should be replaced with more energetic play [7, 8]. These guidelines highlight the importance of, not only motivating for MVPA, but also reducing sitting time, to achieve greater health benefits.

\section{Research gaps}

No study included in this review assessed the movement behaviors that make up the whole day (sleep, sedentary behavior and physical activity of all intensities)[38], which are known to interact, conflate and influence health, ignoring their potential synergic effects on cardiovascular disease development[39]. Therefore, examining the evidence of the movement behaviors combination, might be the key to better understand the complexity of the clustering and interactions of these behaviors and its health effects.

To reduce heterogeneity between studies investigating breaks in sitting time or in sedentary behavior, the new standardized operational definitions of sedentary bouts and breaks proposed by Tremblay and colleagues [1] should be adopted by the research community in this field.

To understand at what level sedentary time and its interruptions are associated with markers of cardiometabolic risk in pediatric populations, more epidemiological studies with objectively measured sedentary breaks in sitting/sedentary time are required. Also, researchers could explore differences between age groups, i.e. early years separately to adolescents.

\section{Strengths and Limitations}

The main strength of this systematic review is the fact that is the first, to our knowledge, to summarize the associations between breaks in sitting time and cardiovascular health outcomes in children and adolescents. This review included a total of 9116 participants, which is a large number considering that only included sixteen studies.

Nonetheless, our review is not without limitations. Due to heterogeneity of the data, metaanalyses were not possible and, therefore, narrative syntheses were conducted.

\section{Conclusion and suggestions}

The association between breaks in sitting time and cardiovascular health outcomes in children is inconsistent and has revealed mostly favorable 


\section{Sousa-Sá E et al.,/2020}

and some null associations. As it is known that there is a negative association between breaks in sitting time and cardiovascular health in adults and given the few studies included in this systematic review, more epidemiological evidence, on the relationship between patterns of sedentary time and markers of cardiovascular health is required to draw definitive conclusions on the association between breaks and cardiovascular health in youth. This will help to inform lifestyle interventions and public health policies intended to diminish chronic disease risk in children and youth, consequently translating into long-term implications on population health.

\section{Supplementary information}

Supplementary information contains the Search strategy.

\section{Authors ORCID ID details}

Sousa-Sá, E.

https://orcid.org/0000-0001-8753-6817

Pereira, JR

https://orcid.org/0000-0001-5491-4316

Zhang, Z

https://orcid.org/0000-0001-6873-7410

Okely, AD

https://orcid.org/0000-0002-1626-8170

Santos, R

https://orcid.org/0000-0002-7604-5753

\section{References}

[1] M.S. Tremblay, S. Aubert, J.D. Barnes, T.J. Saunders, V. Carson, A.E. Latimer-Cheung et al. Sedentary Behavior Research Network (SBRN)-Terminology Consensus Project process and outcome, International Journal of Behavioral Nutrition and Physical Activity, 14 (2017) 75.

[2] WHO. Global Status Report on Noncommunicable Diseases 2014: World Health Organization2014.
[3] W. Bao, S.A. Threefoot, S.R. Srinivasan, G.S. Berenson, Essential hypertension predicted by tracking of elevated blood pressure from childhood to adulthood: the Bogalusa Heart Study, American Journal of Hypertension, 8 (1995) 657-65.

[4] X. Chen, Y. Wang, Tracking of blood pressure from childhood to adulthood: a systematic review and meta-regression analysis, Circulation,117 (2008) 3171-3180.

[5] V.J. Poitras, C.E. Gray, X. Janssen, S. Aubert, V. Carson, G. Faulkner, G.S. Goldfield, J.J. Reilly, M. Sampson , M.S. Tremblay, Systematic review of the relationships between sedentary behaviour and health indicators in the early years (0-4 years), BMC Public Health, 17 (2017) 868.

[6] V. Carson, S. Hunter, N. Kuzik, C.E. Gray, V.J. Poitras, J.P Chaput, T.J. Saunders, P.T. Katzmarzyk, A.D. Okely, S. Connor Gorber, M.E. Kho, M. Sampson, H. Lee, M.S. Tremblay, Systematic review of sedentary behaviour and health indicators in school-aged children and youth: an update, Applied Physiology, Nutrition, and Metabolism, 41 (2016) S240S65.

[7] A.D. Okely, D. Ghersi, K.D. Hesketh, R. Santos, S.P. Loughran, D.P. Cliff, T. Shilton, D. Grant, R.A. Jones, R.M. Stanley, J. Sherring, T. Hinkley, S.G. Trost, C. McHugh, S. Eckermann, K. Thorpe, K. Waters, T.S. Olds, T. Mackey, R. Livingstone, H. Christian, H. Carr, A. Verrender, J.R. Pereira, Z. Zhang, K.L. Downing, M.S. Tremblay, A collaborative approach to adopting/adapting guidelinesThe Australian 24-Hour Movement Guidelines for the early years (Birth to 5 years): an integration of physical activity, sedentary behavior, and sleep, BMC Public Health, 17 (2017) 869.

[8] M.S. Tremblay, J-P. Chaput, K.B. Adamo, S. Aubert, J.D. Barnes, L. Choquette, M. Duggan, G. Faulkner, G.S. Goldfield, C.E. Gray, R. Gruber, K. Janson, I. Janssen, X. Janssen, A. Jaramillo Garcia, N. Kuzik, C. LeBlanc, J. 


\section{Sousa-Sá E et al.,/2020}

MacLean, A.D. Okely, V.J. Poitras, M.E. Rayner, J.J. Reilly, M. Sampson, J.C. Spence, B.W. Timmons, V. Carson, Canadian 24-Hour Movement Guidelines for the Early Years (04 years): An Integration of Physical Activity, Sedentary Behaviour, and Sleep, BMC Public Health, 17 (2017) 874.

[9] D.W. Dunstan, B.A. Kingwell, R. Larsen, G.N. Healy, E. Cerin, M.T. Hamilton, J.E. Shaw, D.A. Bertovic, P.Z. Zimmet, J. Salmon, N. Owen, Breaking up prolonged sitting reduces postprandial glucose and insulin responses, Diabetes Care, 35 (2012) 976-83.

[10] N .Gupta, M. Heiden, M. Aadahl, M. Korshøj, M.B. Jørgensen, A. Holtermann, What is the effect on obesity indicators from replacing prolonged sedentary time with brief sedentary bouts, standing and different types of physical activity during working days? A cross-sectional accelerometer-based study among blue-collar workers, PLoS One, 11 (2016) e0154935.

[11] G.N. Healy, D.W. Dunstan, J. Salmon, E. Cerin, J.E. Shaw, P.Z. Zimmet, N. Owen, Breaks in sedentary time: beneficial associations with metabolic risk, Diabetes Care, 31 (2008) 661666.

[12] G.N. Healy, C.E. Matthews, D.W. Dunstan, E.A. Winkler, N. Owen, Sedentary time and cardiometabolic biomarkers in US adults: NHANES 2003-06, European Heart Journal, 32 (2011) 590-597.

[13] P.T. Katzmarzyk, Standing and mortality in a prospective cohort of Canadian adults, Medicine \& Science in Sports \& Exercise, 46 (2014) 940-946.

[14] D. Moher, A. Liberati, J. Tetzlaff, D.G. Altman, Preferred reporting items for systematic reviews and meta-analyses: the PRISMA statement, Annals of Internal Medicine, 151 (2009) 264-269.

[15] C. Schardt, M.B. Adams, T. Owens, S. Keitz, P. Fontelo, Utilization of the PICO framework to improve searching PubMed for clinical questions, BMC Medical Informatics and Decision Making, 7 (2007) 1.

[16] G.H. Guyatt, A.D. Oxman, H.J. Schünemann, P. Tugwell, A. Knottnerus, GRADE guidelines: a new series of articles in the Journal of Clinical Epidemiology, Journal of Clinical Epidemiology, 64 (2011) 380-382.

[17] G.H. Guyatt, A.D. Oxman, G. Vist, R. Kunz, J. Brozek, P. Alonso-Coello, V. Montori , E.A. Akl , B. Djulbegovic , Y. Falck-Ytter , S.L. Norris , J.W. Jr Williams , D. Atkins , J. Meerpohl , H.J. Schünemann, GRADE guidelines: 4 . Rating the quality of evidence-study limitations (risk of bias), Journal of Clinical Epidemiology, 64 (2011) 407-415.

[18] T.J. Saunders, J-P. Chaput, G.S. Goldfield, R.C. Colley, G.P. Kenny, E. Doucet, M.S. Tremblay, Prolonged sitting and markers of cardiometabolic disease risk in children and youth: a randomized crossover study, Metabolism, 62(2013) 1423-1428.

[19] Saunders TJ, Tremblay MS, Mathieu M-È, Henderson M, O'Loughlin J, Tremblay A et al. Associations of sedentary behavior, sedentary bouts and breaks in sedentary time with cardiometabolic risk in children with a family history of obesity, PLoS One, 8 (2013) e79143.

[20] R.C. Colley, D. Garriguet, I. Janssen, S.L. Wong, T.J. Saunders, V. Carson, M.S. Tremblay, The association between accelerometermeasured patterns of sedentary time and health risk in children and youth: results from the Canadian Health Measures Survey, BMC Public Health, 13 (2013) 200.

[21] V. Carson, M. Stone, G. Faulkner, Patterns of Sedentary Behavior and Weight Status Among Children, Pediatric Exercise Science, 26 (2014) 95-102.

[22] T.J. Saunders, J-P. Chaput, G.S. Goldfield, R.C. Colley, G.P. Kenny, E. Doucet, M.S. Tremblay, Children and youth do not compensate for an imposed bout of prolonged sitting by reducing subsequent food intake or 


\section{Sousa-Sá E et al.,/2020}

increasing physical activity levels: a randomised cross-over study, The British Journal of Nutrition, 111 (2014) 747-754.

L. Gabel, N.D. Ridgers, P.A. Della Gatta, L. Arundell, E. Cerin, S. Robinson, R.M. Daly, D.W. Dunstan, J. Salmon, Associations of sedentary time patterns and TV viewing time with inflammatory and endothelial function biomarkers in children, Pediatric Obesity, 11 (2016) 194-201.

[24] A.M. Contardo-Ayala, J. Salmon, A. Timperio, B. Sudholz, N.D. Ridgers, P. Sethi, D.W. Dunstan, Impact of an 8-Month Trial Using Height-Adjustable Desks on Children's Classroom Sitting Patterns and Markers of [30] Cardio-Metabolic and Musculoskeletal Health, International Journal of Environmental Research and Public Health, 13 (2016).

[25] A.M. Contardo-Ayala, B. Sudholz, J. Salmon, D.W. Dunstan, N.D. Ridgers, L. Arundell, A. Timperio, The impact of height-adjustable desks and prompts to break-up classroom [31] sitting on adolescents' energy expenditure, adiposity markers and perceived musculoskeletal discomfort, PLoS One, 13 (2018).

[26] E. Sousa-Sá, J.R. Pereira, Z. Zhang, S.L.C. [32] Veldman, A.D. Okely, R. Santos, Association between breaks in sitting time and adiposity in Australian toddlers: Results from the GETUP! Study, Scandinavian Journal of Medicine \& Science in Sports, 29 (2019) 259-265.

[27] V. Carson, I. Janssen, Volume, patterns, and types of sedentary behavior and cardiometabolic health in children and adolescents: a cross-sectional study, BMC Public Health, 11 (2011) 274.

[28] B.R. Belcher, D. Berrigan, A. Papachristopoulou, S.M. Brady, S.B. Bernstein, R.J. Brychta, J.D. Hattenbach, Tigner IL Jr, A.B. Courville, B.E. Drinkard, K.P. Smith, D.R. Rosing, P.L. Wolters, Chen KY, J.A. Yanovski, Effects of Interrupting Children's Sedentary Behaviors With Activity on Metabolic Function: A Randomized Trial, The Journal Of
Clinical Endocrinology And Metabolism, 100 (2015) 3735-3743.

M.M. Broadney, B.R. Belcher, D.A. Berrigan, R.J. Brychta, I.L. Tigner, F. Shareef, A. Papachristopoulou, J.D. Hattenbach, E.K. Davis, S.M. Brady, S.B. Bernstein, A.B. Courville, B.E. Drinkard, K.P. Smith, D.R. Rosing, P.L. Wolters, K.Y. Chen, J.A. Yanovski, Effects of interrupting sedentary behavior with short bouts of moderate physical activity on glucose tolerance in children with overweight and obesity: A randomized crossover trial, Diabetes Care, 41 (2018) 2220-2228.

D.P. Bailey, S.J. Charman, T. Ploetz, L.A. Savory, C.J. Kerr, Associations between prolonged sedentary time and breaks in sedentary time with cardiometabolic risk in 10-14-year-old children: The HAPPY study, Journal of Sports Sciences, 35 (2017) 21642171.

K.P. Dowd, D.M. Harrington, A. Hannigan, A.E. Donnelly, Light-Intensity Physical Activity Is Associated with Adiposity in Adolescent Females, Medicine \& Science in Sports \& Exercise, 46 (2014) 2295-300.

P.B. Júdice, A.M. Silva, J. Berria, E.L. Petroski, U. Ekelund, L.B. Sardinha, Sedentary patterns, physical activity and health-related physical fitness in youth: a cross-sectional study, International Journal of Behavioral Nutrition \& Physical Activity, 14 (2017) 1-10.

S.G. Trost, B.S. Fees, S.J. Haar, A.D. Murray, L.K. Crowe, Identification and validity of accelerometer cut - points for toddlers, Obesity, 20 (11) 2317-2319.

[34] E. Johansson, L-M. Larisch, C. Marcus, M. Hagströmer, Calibration and validation of a wrist-and hip-worn ActiGraph accelerometer in 4-year-old children, PLoS One,11 (2016) e0162436.

[35] X. Janssen, D.P. Cliff, J.J. Reilly, T. Hinkley, R.A. Jones, M. Batterham,U. Ekelund, S. Brage, A.D. Okely, Validation of activPAL defined 
sedentary time and breaks in sedentary time [38] in 4-to 6-year-olds, Pediatric Exercise Science, 26 (2014) 110-117.

[36] T. Altenburg, M. De Niet, M. Verloigne, I. De Bourdeaudhuij, O. Androutsos, Y. Manios, E. Kovacs, B. Bringolf-Isler, J. Brug, M. Chinapaw, [39] Occurrence and duration of various operational definitions of sedentary bouts and cross-sectional associations with cardiometabolic health indicators: the ENERGY-project, Preventive Medicine, 71 (2015) 101-106.

[37] N. Cavill, S. Biddle, J.F. Sallis, Health enhancing physical activity for young people: Statement of the United Kingdom Expert Consensus Conference, Pediatric Exercise Science, 13 (2001) 12-25.
M.S. Tremblay, R.C. Colley, T.J. Saunders, G.N. Healy, N. Owen, Physiological and health implications of a sedentary lifestyle, Applied Physiology, Nutrition, and Metabolism, 35 (2010) 725-740.

S.F. Chastin, J. Palarea-Albaladejo, M.L. Dontje, D.A. Skelton, Combined effects of time spent in physical activity, sedentary behaviors and sleep on obesity and cardio-metabolic health markers: a novel compositional data analysis approach, PLoS One,10 (2015) e0139984.

\section{Study registration}

PROSPERO - International Prospective Register of Systematic Review: CRD42018083790.

\section{Funding and Acknowledgement}

E Sousa-Sá, JR Pereira and J McNeill are supported by PhD scholarships from University of Wollongong. Z Zhang is supported by a PhD scholarship from China Scholarship Council and an International Postgraduate Tuition Award from University of Wollongong. R Santos was supported by a Discovery Early Career Research Award from the Australian Research Council (DE150101921)

\section{Conflict of interest}

None of the authors have any conflicts of interest to declare.

\section{About The License}

\section{()ㅜ)}

Attribution 4.0 International (CC BY 4.0)
The text of this article is licensed under a Creative Commons Attribution 4.0 International License 